\title{
A high-fructose diet impairs basal and stress-mediated lipid metabolism in healthy male subjects
}

\author{
Andrew Abdel-Sayed, Christophe Binnert, Kim-Anne Lê, Murielle Bortolotti, Philippe Schneiter \\ and Luc Tappy* \\ Department of Physiology, School of Biology and Medicine, University of Lausanne, 7 rue du Bugnon, Lausanne CH-1005, \\ Switzerland
}

(Received 19 July 2007 - Revised 21 November 2007 - Accepted 21 November 2007)

The effects of a $7 \mathrm{~d}$ high-fructose diet (HFrD) or control diet on lipid metabolism were studied in a group of six healthy lean males. Plasma NEFA and $\beta$-hydroxybutyrate concentrations, net lipid oxidation (indirect calorimetry) and exogenous lipid oxidation $\left({ }^{13} \mathrm{CO}_{2}\right.$ production) were monitored in basal conditions, after lipid loading (olive oil labelled with $\left[{ }^{13} \mathrm{C}\right]$ triolein) and during a standardised mental stress. Lactate clearance and the metabolic effects of an exogenous lactate infusion were also monitored. The HFrD lowered plasma concentrations of NEFA and $\beta$-hydroxybutyrate as well as lipid oxidation in both basal and after lipid-loading conditions. In addition, the HFrD blunted the increase in plasma NEFA and exogenous lipid oxidation during mental stress. The HFrD also increased basal lactate concentrations by $31.8 \%$, and lactate production by $53.8 \%$, while lactate clearance remained unchanged. Lactate infusion lowered plasma NEFA with the control diet, and net lipid oxidation with both the HFrD and control diet. These results indicate that a $7 \mathrm{~d} \mathrm{HFrD} \mathrm{markedly} \mathrm{inhibits} \mathrm{lipolysis} \mathrm{and} \mathrm{lipid} \mathrm{oxidation.} \mathrm{The} \mathrm{HFrD} \mathrm{also} \mathrm{increases}$ lactate production, and the ensuing increased lactate utilisation may contribute to suppress lipid oxidation.

High-fructose diets: Lipid metabolism: Lactate kinetics

Consumption of fructose-containing sweetened beverages has increased markedly over the past decades, and is thought to play a significant role in the current epidemics of obesity and metabolic disorders ${ }^{(1,2)}$. In rodents, high-sucrose or -fructose diets cause obesity, insulin resistance, diabetes mellitus, dyslipidaemia and high blood pressure ${ }^{(3)}$, while in humans, high-fructose hyperenergetic diets (HFrD) lead, over a few days, to the development of hypertriacylglycerolaemia and hepatic insulin resistance ${ }^{(4-8)}$. The metabolic effects of fructose have been widely studied, as reported in several recent reviews ${ }^{(9,10)}$; however, the mechanisms leading to fructoseinduced insulin resistance remain poorly understood.

Several observations indicate that diet-induced insulin resistance bears strong relationships with disturbances of intracellular lipid metabolism ${ }^{(11)}$. An impaired capacity to oxidise lipids in skeletal muscle, and possibly in other tissues, is present in insulin-resistant individuals ${ }^{(12,13)}$. Impaired fat oxidation may in turn lead to the accumulation of so-called 'ectopic' lipids, i.e. intracellular storage of TAG in non-adipose tissue cells, and of lipid metabolites, which may interfere with insulin signalling and its actions ${ }^{(14)}$. It can therefore be suspected that inhibition of lipid oxidation may participate in the adverse metabolic effects of high-fructose diets. To evaluate this hypothesis, we studied the effects of a 6-7 d HFrD on lipid metabolism, in a group of healthy young males. Whole-body net fat oxidation, exogenous fat oxidation, adipose tissue lipolysis and plasma ketone body release were challenged by a lipid load that was followed by a mental stress, which stimulates sympathetic activity ${ }^{(15)}$. All parameters were measured under fasting conditions, after lipid loading and after mental stress. Since an HFrD also causes a substantial rise in plasma lactate concentrations $^{(6)}$, and increased lactate utilisation may in turn affect lipid metabolism ${ }^{(16-18)}$, we also studied lactate kinetics with $\left[{ }^{13} \mathrm{C}_{3}\right]$ lactate infusion in the same subjects.

\section{Methods \\ Subjects}

Six healthy young males (age 24.7 (SD 3.1) years; weight 78.3 (SD 7.4) $\mathrm{kg}$; BMI 23.1 (SD 2.2) $\mathrm{kg} / \mathrm{m}^{2}$ ) were recruited into the study. All were in good health, had no family history of type 2 diabetes mellitus and were non-smokers. None took any medication at the time of inclusion or during the study. The experimental protocol was approved by the ethical board of Lausanne University School of Biology and Medicine, and every subject provided informed, written consent.

\section{Dietary intervention}

Each subject was randomised to one of two diets: (1) a $7 d$ control diet calculated to provide his predicted energy 
requirements ${ }^{(19)}$ multiplied by a factor of 1.5 to account for the thermic effect of food and sedentary activity that was composed of $55 \%$ carbohydrate, $30 \%$ fat and $15 \%$ protein or (2) the same diet supplemented with $3.0 \mathrm{~g}$ fructose $/ \mathrm{kg}$ body weight per $\mathrm{d}$, corresponding to the fructose content of about 4 litres sweetened beverage. This resulted in a mean extra fructose intake of $234 \mathrm{~g}$ and an excess energy intake of about $3640 \mathrm{~kJ} / \mathrm{d}$, resulting in an overall diet providing $67 \%$ carbohydrate, $22 \%$ fat and $11 \%$ protein. The study was performed on an out-patient basis, and fructose was provided as packs of fructose monohydrate to be dissolved in water and to be drunk in three divided doses with each main meal. A 3-5-week wash-out period separated the two diets. We have observed in previous studies that a wash-out period of this duration is sufficient for complete return of metabolic parameters to baseline ${ }^{(6,7)}$.

\section{Metabolic study 1: lactate infusion}

On the morning of day 6 of each diet, subjects reported to the metabolic investigation unit at 07.00 hours after an overnight fast. Upon arrival they were requested to void, after which their body weight was recorded. The subject was then comfortably installed in a bed in a reclining position where they remained quietly watching television for the next $6 \mathrm{~h}$. Two venous catheters were inserted, one into a forearm vein for infusion of $\left[6,6-{ }^{2} \mathrm{H}_{2}\right]$ glucose $(2 \mathrm{mg} / \mathrm{kg}$ bolus followed by $20 \mu \mathrm{g} / \mathrm{kg}$ per min between time -120 to time $180 \mathrm{~min}$ ) and exogenous lactate $(0.5 \mathrm{M}$-sodium lactate labelled with $0.05 \%$ $\left[{ }^{13} \mathrm{C}_{3}\right]$ lactate, infused at a rate of $20 \mathrm{mmol} / \mathrm{kg}$ per min between time 0 and $180 \mathrm{~min}$ ), and the other into a wrist vein of the contralateral arm for blood collections. The hand on this side was maintained inside a thermostabilised box heated at $50^{\circ} \mathrm{C}$ to achieve arterialisation of venous blood. Respiratory gas exchange was measured continuously using a ventilated hood, open-circuit indirect calorimetry (Deltatrac II; Datex Instruments, Helsinki, Finland). Blood collections were obtained at time -120 and every $30 \mathrm{~min}$ between -30 and $180 \mathrm{~min}$ for hormone and substrate measurements and for the determination of plasma $\left[6,6-{ }^{2} \mathrm{H}_{2}\right]$ glucose enrichment. End-expiratory breath samples were collected every $30 \mathrm{~min}$ in vacutainers for determination of ${ }^{13} \mathrm{CO}_{2}$ enrichment.

A timed urine collection was taken during the experiment to determine urea $\mathrm{N}$ excretion rate.

\section{Metabolic study 2: lipid loading and mental stress}

The present study took place on day 8 of each diet, and instrumentation procedures were the same as described for study 1 . After $120 \mathrm{~min}$ of fasting measurements, three oral loads of $10 \mathrm{~g}$ olive oil labelled with $100 \mathrm{mg}\left[1-{ }^{13} \mathrm{C}_{3}\right]$ triolein (Cambridge Isotope Laboratory, Cambridge, MA, USA) were ingested at times 0,60 and $120 \mathrm{~min}$ to measure exogenous fat oxidation ${ }^{(20)}$. A mental stress procedure, consisting of 5 min periods of complex mental arithmetic alternated with 5 min periods of Stroop's word colour conflict tests ${ }^{(15)}$, was applied between 180 and $210 \mathrm{~min}$. This procedure is known to activate the sympatho-adrenal axis and to produce stimulation of lipolysis $^{(21,22)}$. Blood collections were obtained at $-120 \mathrm{~min}$ and every $30 \mathrm{~min}$ from -30 to $180 \mathrm{~min}$, and subsequently at 190,200, 210 and $240 \mathrm{~min}$ for hormone and substrate measurements and for determination of plasma $\left[6,6-{ }^{2} \mathrm{H}_{2}\right]$ glucose enrichment. Saliva samples were obtained in basal conditions, after lipid loading and every $10 \mathrm{~min}$ during the $30 \mathrm{~min}$ mental stress test by using a saliva collection kit (Sarstedt AG, Sevelen, Switzerland). Expiratory breath samples were collected every $30 \mathrm{~min}$ in vacutainers for determination of ${ }^{13} \mathrm{CO}_{2}$ enrichment.

A timed urine collection was performed during the experiment to determine urea $\mathrm{N}$ excretion.

\section{Analytical procedures}

Plasma glucose was measured with the glucose oxidase method, using a Beckman Glucose Analyzer II (Beckman Instruments, Palo Alto, CA, USA). Plasma insulin and salivary cortisol concentrations were measured using RIA kits (Biodata, Guidoni, Montecello, Italy and DPC Inc., Los Angeles, CA, USA, respectively). Plasma NEFA were measured colorimetrically using a kit from Wako (Freiburg, Germany). Plasma $\beta$-hydroxybutyrate and TAG were measured enzymically using a kit from Boehringer (Mannheim, Germany). Plasma adrenaline and noradrenaline were measured by HPLC ${ }^{(23)}$. Plasma $\left[6,6-{ }^{2} \mathrm{H}_{2}\right]$ glucose isotopic enrichment was measured with GC-MS, as described previously ${ }^{(24,25)}$. Breath ${ }^{13} \mathrm{CO}_{2}$ isotopic enrichment was measured by isotope ratio MS on a Tracermass C/N (SerCon Ltd, Crewe, Cheshire, UK).

\section{Calculations}

All calculation procedures have been reported in detail elsewhere. The rates of glucose appearance and disappearance were calculated from plasma $\left[6,6-{ }^{2} \mathrm{H}_{2}\right]$ glucose isotopic enrichment using Steele's equations for steady-state conditions ${ }^{(26)}$. Exogenous oleate $^{(20)}$ and lactate ${ }^{(27)}$ oxidations were calculated from ${ }^{13} \mathrm{CO}_{2}$ production. Whole-body net substrate oxidation rates were calculated using the equations of Livesey \& Elia $^{(28)}$. During lactate infusion, it was assumed that lactate disappearance was equal to net lactate oxidation in order to calculate net glucose and lipid oxidation ${ }^{(27,29)}$. This net lactate oxidation may include oxidation of glucose synthesised from lactate. Lactate clearance was calculated using a 'pharmacokinetic' model from changes in plasma lactate concentrations during exogenous lactate infusion, and basal lactate production was calculated as basal lactate concentration/lactate clearance $^{(30)}$.

\section{Statistical analysis}

All results in the text and tables are expressed as mean values with their standard errors. Comparisons between the control diet and the HFrD, or between fasting conditions, post-lipid conditions, and mental stress were done with the Wilcoxon signed rank test.

\section{Results}

Lipid metabolism in basal and after lipid-loading conditions is presented in Table 1 and illustrated in Fig. 1.

In basal conditions, the HFrD, compared with the control diet, decreased plasma NEFA by $19.5 \%$, plasma $\beta$-hydroxybutyrate by $78.2 \%$ and whole-body lipid oxidation by 
Table 1. Effects of a high-fructose diet (HFrD) on lipid and energy metabolism in basal conditions and after lipid loading* (Mean values with their standard errors)

\begin{tabular}{|c|c|c|c|c|c|c|c|c|}
\hline & \multicolumn{4}{|c|}{ Control } & \multicolumn{4}{|c|}{ HFrD } \\
\hline & \multicolumn{2}{|c|}{ Basal } & \multicolumn{2}{|c|}{ Lipid loading } & \multicolumn{2}{|c|}{ Basal } & \multicolumn{2}{|c|}{ Lipid loading } \\
\hline & Mean & SEM & Mean & SEM & Mean & SEM & Mean & SEM \\
\hline Plasma glucose (mmol/l) & $4 \cdot 6$ & 0.1 & $4 \cdot 3$ & 0.2 & 4.5 & 0.1 & 4.5 & $0 \cdot 1$ \\
\hline Plasma NEFA (mmol/l) & 0.41 & 0.09 & 0.45 & 0.05 & $0.33 \dagger$ & 0.08 & 0.41 & 0.06 \\
\hline Plasma TAG (mmol/l) & 0.49 & 0.05 & 0.67 & $0 \cdot 11$ & $0.81 \dagger$ & $0 \cdot 18$ & $1.00 \dagger$ & 0.20 \\
\hline Plasma $\beta$-hydroxybutyrate (mmol/l) & 55 & 17 & & & $13+$ & 3 & & \\
\hline Energy expenditure (kJ/kg per min) & 0.057 & 0.005 & 0.057 & 0.004 & 0.057 & 0.005 & 0.057 & 0.005 \\
\hline Carbohydrate oxidation (mg/kg per min) & $1 \cdot 24$ & 0.19 & 0.92 & $0 \cdot 14$ & $1.99 \dagger$ & $0 \cdot 19$ & $1.62 \dagger$ & 0.17 \\
\hline Lipid oxidation (mg/kg per min) & 0.71 & $0 \cdot 11$ & 0.89 & $0 \cdot 10$ & $0.49 \dagger$ & $0 \cdot 10$ & $0.73 \dagger$ & 0.05 \\
\hline Exogenous lipid oxidation & & & 27 & 3 & & & 24 & 1 \\
\hline
\end{tabular}

* Basal conditions: blood samples were obtained in the fasting state, with a rest period of $120 \mathrm{~min}$ after instrumentation of the subject. Lipid loading: blood samples were collected 150 and $180 \mathrm{~min}$ after lipid loading, and the values obtained were averaged for presentation. $\dagger$ Mean value was significantly different from that of control $(P<0.05)$.

$21.3 \%$ (all $P<0.05$; Table 1). After lipid loading, there was no significant change in plasma glucose, insulin or NEFA concentrations in either condition but plasma $\beta$-hydroxybutyrate increased $(P<0.05)$ in both conditions, although it tended to be lower with the HFrD $(P=0 \cdot 12$; Fig. 1$)$. Whole-body net lipid oxidation increased slightly by $0.18 \mathrm{mg} / \mathrm{kg}$ per $\mathrm{min}$ with the control diet and by $0.24 \mathrm{mg} / \mathrm{kg}$ per min with the HFrD (NS). Absolute levels of lipid oxidation remained lower $(P<0 \cdot 05)$ on the HFrD than with the control diet (Table 1). Breath ${ }^{13} \mathrm{CO}_{2}$ isotopic enrichment and oxidation of exogenous lipids (Table 1) over the first $3 \mathrm{~h}$ after lipid loading were comparable between the HFrD and control diet.
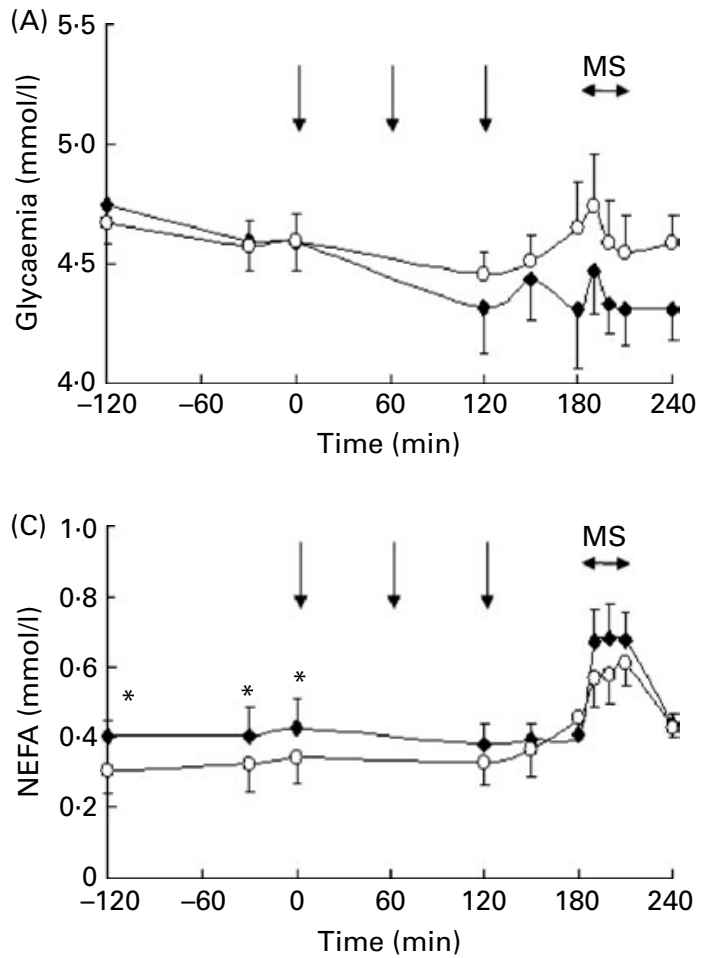

\section{Lipid metabolism during mental stress}

During mental stress, plasma cortisol, adrenaline and noradrenaline concentrations increased to the same extent with the HFrD and with the control diet (Table 2), while glucose and insulin concentrations remained unchanged (Fig. 1). Plasma NEFA increased significantly during mental stress, but the integrated incremental area under the curve was significantly lower with the HFrD $(P<0 \cdot 03)$ than with the control diet (Fig. 1). The concentration of $\beta$-hydroxybutyrate did not change during mental stress, and tended to be lower with the HFrD than with the control diet $(P=0 \cdot 12$; Fig. 1). During the
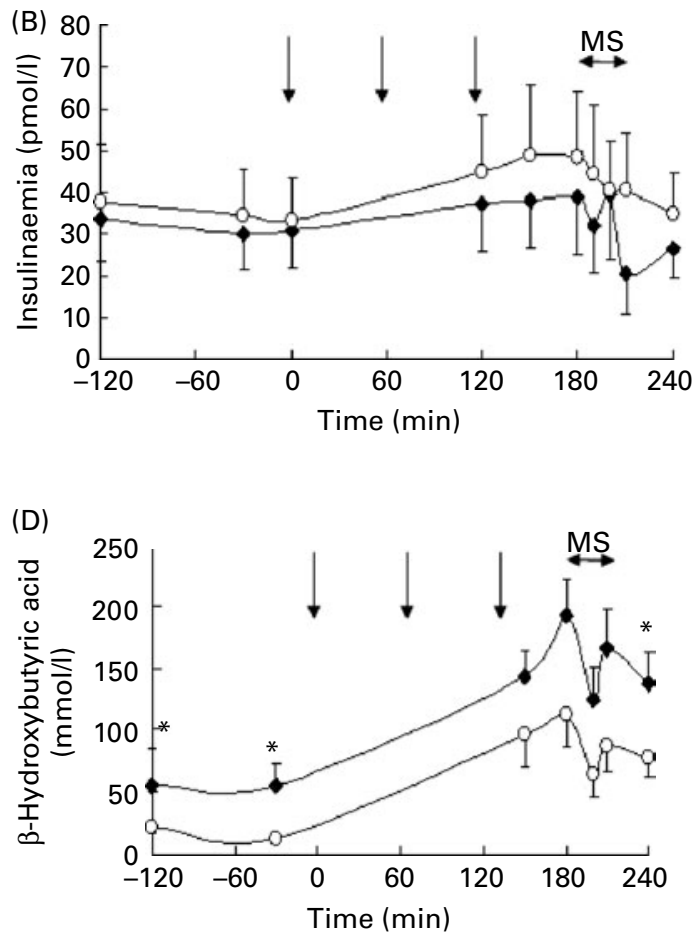

Fig. 1. Changes in (A) plasma glucose, (B) insulin, (C) NEFA and (D) $\beta$-hydroxybutyrate concentrations during lipid loading and during mental stress (MS). Values are means, with standard errors represented by vertical bars. $(\bigcirc)$, High-fructose diet; $(\bullet)$, control conditions; $\downarrow$, times at which olive oil was ingested. ${ }^{*}$ Mean values were significantly different $(P<0.05)$. 
A. Abdel-Sayed et al.

Table 2. Effects of a high-fructose diet (HFrD) on lipid and energy metabolism during mental stress (Mean values with their standard errors)

\begin{tabular}{|c|c|c|c|c|}
\hline & \multicolumn{2}{|c|}{ Control } & \multicolumn{2}{|c|}{ HFrD } \\
\hline & Mean & SEM & Mean & SEM \\
\hline \multicolumn{5}{|l|}{ Baseline* } \\
\hline Glucose (mmol/l) & $4 \cdot 3$ & 0.2 & $4 \cdot 7$ & $0 \cdot 2$ \\
\hline NEFA $(\mathrm{mmol} / \mathrm{l})$ & 0.41 & 0.06 & 0.46 & 0.05 \\
\hline Adrenaline $(\mathrm{pg} / \mathrm{ml})$ & 47 & 7 & 46 & 13 \\
\hline Noradrenaline $(\mathrm{pg} / \mathrm{ml})$ & 130 & 55 & 135 & 65 \\
\hline Salivary cortisol (nmol/l) & $4 \cdot 1$ & 0.4 & 4.6 & 0.2 \\
\hline \multicolumn{5}{|l|}{$\begin{array}{l}\text { Incremental areas under the curve } \\
\text { after mental stress }\end{array}$} \\
\hline Glucose $(\mathrm{mmol} / \mathrm{l} \times 30 \mathrm{~min})$ & 16 & 25 & -9 & 8 \\
\hline $\mathrm{NEFA}(\mathrm{mmol} / \mathrm{l} \times 30 \mathrm{~min})$ & 8.0 & $2 \cdot 2$ & $3.8 \dagger$ & $2 \cdot 4$ \\
\hline Adrenaline $(\mathrm{pg} / \mathrm{ml} \times 30 \mathrm{~min})$ & 689 & 235 & 620 & 346 \\
\hline Noradrenaline $(\mathrm{pg} / \mathrm{ml} \times 30 \mathrm{~min})$ & 2783 & 562 & 2746 & 850 \\
\hline Salivary cortisol (nmol/l × $30 \mathrm{~min})$ & 11.4 & $5 \cdot 3$ & 32 & 23 \\
\hline $\begin{array}{l}\text { Exogenous lipid oxidation after mental } \\
\text { stress }(\mathrm{mg} / \mathrm{kg} \text { per } \mathrm{h})\end{array}$ & 15 & 2 & $11 \dagger$ & 2 \\
\hline
\end{tabular}

*Basal conditions: blood samples were obtained immediately before mental stress, i.e. $180 \mathrm{~min}$ after the beginning of the lipid loading $\dagger$ Mean value was significantly different from that of control $(P<0.05)$.

60 min period between 180 and $240 \mathrm{~min}$, which corresponded to the $30 \mathrm{~min}$ mental stress $+30 \mathrm{~min}$ recovery, breath ${ }^{13} \mathrm{CO}_{2}$ and exogenous lipid oxidation increased with both the HFrD and control diet, but were significantly lower after the HFrD $(P<0 \cdot 05$; Table 1$)$.

\section{Lactate metabolism}

Basal lactate production increased by $52.4 \%$ while on the HFrD $(P<0.001)$. At the end of the dietary periods basal lactate concentrations were $33.2 \%$ higher with the HFrD than with the control diet $(P<0 \cdot 01)$ (Table 3 and Fig. 2), but the increment in plasma lactate (Fig. 2), and hence calculated lactate clearance (Table 3), were comparable between the two conditions. Calculated basal lactate production was significantly higher while on the HFrD.

Exogenous lactate infusion did not change glucose rate of appearance or plasma glucose concentrations (Table 3).
Plasma NEFA tended to be decreased $(-15 \% ; P=0 \cdot 12)$ after the control diet, but not after the HFrD (Fig. 2 and Table 3). Lactate infusion decreased net whole-body lipid oxidation by $30.7 \%$ with the control diet $(P<0.02)$ and $34.1 \%$ with the HFrD $(P<0 \cdot 01$; Table 3$)$. Breath ${ }^{13} \mathrm{CO}_{2}$ isotopic enrichment increased similarly after the HFrD and control diet and exogenous lactate oxidation was similar under both conditions (8.0 (SEM 0.4) $\mu \mathrm{mol} / \mathrm{kg}$ per min after the control diet v. 8.7 (SEM 0.5) $\mu \mathrm{mol} / \mathrm{kg}$ per min after the HFrD; NS).

\section{Discussion}

The present results demonstrate than a $7 \mathrm{~d}$ HFrD has important effects on lipid metabolism and oxidation in healthy human subjects and caused a significant $(P<0.05)$ increase in fasting plasma TAG concentrations ${ }^{(4,6,7,31)}$. Although compliance to fructose supplementation was not directly assessed, the consistency of this observation with previous reports $(4,6,7,9,31)$

Table 3. Effects of a high-fructose diet (HFrD) on energy and substrate metabolism in basal conditions and after lactate infusion (Mean values with their standard errors)

\begin{tabular}{|c|c|c|c|c|c|c|c|c|}
\hline & \multicolumn{4}{|c|}{ Control } & \multicolumn{4}{|c|}{ HFrD } \\
\hline & \multicolumn{2}{|c|}{ Basal } & \multicolumn{2}{|c|}{ Lactate } & \multicolumn{2}{|c|}{ Basal } & \multicolumn{2}{|c|}{ Lactate } \\
\hline & Mean & SEM & Mean & SEM & Mean & SEM & Mean & SEM \\
\hline Plasma glucose (mmol/l) & $4 \cdot 8$ & 0.1 & 4.9 & 0.1 & $5 \cdot 1$ & 0.2 & 5.0 & 0.2 \\
\hline Plasma NEFA (mmol/l) & 0.60 & 0.07 & 0.52 & 0.04 & $0.36^{*}$ & 0.09 & $0.39^{*}$ & 0.09 \\
\hline Plasma lactate $(\mathrm{mmol} / \mathrm{l})$ & 0.85 & 0.05 & $2.50 \dagger$ & 0.09 & $1 \cdot 12$ & 0.17 & $2.75 \dagger$ & 0.14 \\
\hline Lactate clearance $(\mathrm{ml} / \mathrm{min})$ & & & 12 & 1 & & & 13 & 2 \\
\hline Lactate production ( $\mu \mathrm{mol} / \mathrm{kg}$ per min) & $10 \cdot 4$ & $1 \cdot 2$ & & & $16 \cdot 0^{*}$ & $5 \cdot 3$ & & \\
\hline Glucose rate of appearance ( $\mu \mathrm{mol} / \mathrm{kg}$ per min) & $11 \cdot 5$ & 0.5 & $10 \cdot 4$ & 0.5 & $11 \cdot 8$ & 0.5 & $10 \cdot 3$ & 0.5 \\
\hline Energy expenditure $(\mathrm{kJ} / \mathrm{kg}$ per $\mathrm{min})$ & 0.057 & 0.005 & 0.062 & 0.005 & 0.054 & 0.005 & 0.057 & 0.004 \\
\hline Carbohydrate oxidation (mg/kg per min) & 1.36 & 0.11 & $0.65 \dagger$ & $0 \cdot 19$ & $1.95^{\star}$ & 0.24 & $0.85^{\star} \dagger$ & 0.25 \\
\hline Lipid oxidation (mg/min) & 0.65 & 0.06 & $0.45 \dagger$ & $0 \cdot 11$ & $0.41^{*}$ & 0.07 & $0.27^{\star} \dagger$ & 0.09 \\
\hline Lactate oxidation ( $\mu \mathrm{mol} / \mathrm{kg}$ per min) & 0 & 0 & 20 & 0 & 0 & 0 & 20 & 0 \\
\hline
\end{tabular}

* Mean value was significantly different from that of control $(P<0.05)$.

$\dagger$ Mean value was significantly different from that in basal conditions $(P<0.05)$. 
(A)

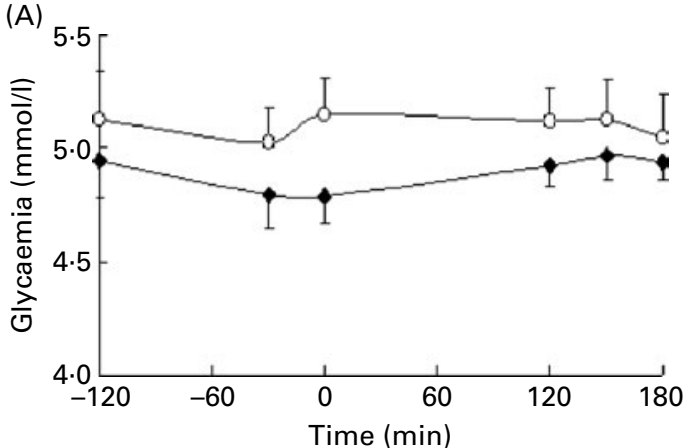

(C)

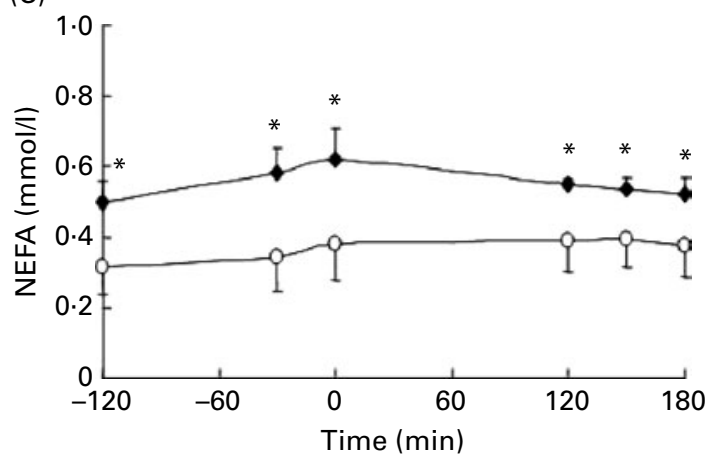

(B)

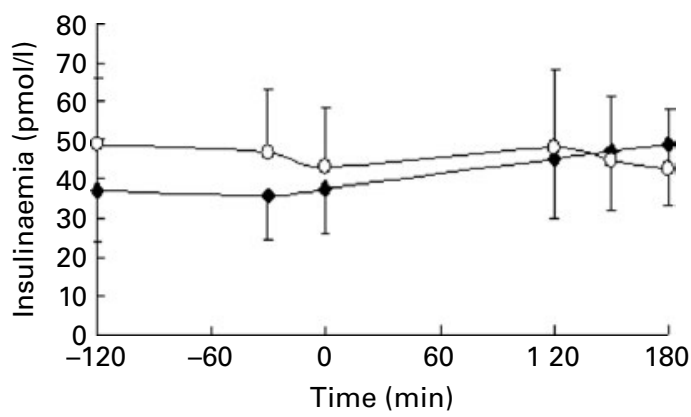

(D)

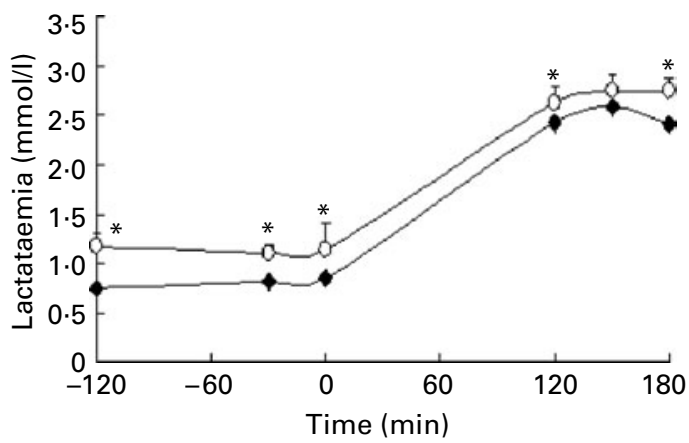

Fig. 2. Changes in (A) plasma glucose, (B) insulin, (C) NEFA and (D) lactate concentrations during exogenous lactate infusion. Values are means, with standard errors represented by vertical bars. $(O)$, High-fructose diet; $(\bullet)$, control conditions. ${ }^{*}$ Mean values were significantly different $(P<0.05)$.

provides indirect evidence that the bulk of the supplementary fructose prescribed to the participants was indeed consumed. Besides this well-known increase in plasma TAG concentrations, the HFrD significantly inhibited several pathways of lipid catabolism. In basal conditions, the HFrD significantly decreased plasma NEFA concentrations, net lipid oxidation and plasma $\beta$-hydroxybutyrate concentrations. After lipid loading, the increase in net lipid oxidation and exogenous lipid oxidation were comparable after the HFrD and control diet, but mental stress markedly blunted the stimulation of plasma NEFA and $\beta$-hydroxybutyrate release. Whole-body net lipid oxidation could not be calculated during mental stress, because the significant degree of hyperventilation which occurs during this procedure precludes the accurate calculation of the $\mathrm{RER}^{(15)}$. Exogenous lipid oxidation could, however, still be calculated since this procedure is less sensitive to small inaccuracies in the measurement of $\mathrm{CO}_{2}$ production ${ }^{(32)}$, and was also significantly inhibited after the HFrD.

The lower basal plasma NEFA concentrations, and the blunted response of NEFA to mental stress, indicate an inhibition of adipose tissue lipolysis after the HFrD. Under such conditions, HFrD-induced hypertriacylglycerolaemia is unlikely to be secondary to an increased hepatic re-esterification, and is likely to be related to fructose-induced stimulation of hepatic de novo lipogenesis. However, the inhibition of lipolysis may in turn be directly responsible for lower whole-body net lipid oxidation and plasma $\beta$-hydroxybutyrate concentrations, since NEFA concentrations are the main determinant of these processes. Alternatively, a direct inhibition of hepatic $\beta$-oxidation and ketogenesis may also be involved. Rats prone to develop diet-induced obesity exhibit similar alterations to those observed in human subjects after the HFrD, i.e. decreased basal whole-body and hepatic lipid oxidation and blunted stimulation of ketone body release. These effects were associated with decreased hepatic expression of genes involved in lipid transport and oxidation ${ }^{(33)}$. Fructose, by exerting a direct effect on liver metabolism, is likely to act on similar pathways. In support of this hypothesis, it has been reported that an HFrD, but not a high-glucose diet, markedly reduces hepatic $\beta$-oxidation in rats through inhibition of the expression of PPAR $\alpha$-dependent genes ${ }^{(34)}$.

Intriguingly, these effects of the HFrD on lipid metabolism were observed in the absence of significant changes in plasma insulin, and with only minimal changes in plasma glucose, suggesting that a change in insulin secretion is not primarily involved. The HFrD therefore lowered plasma NEFA presumably through inhibition of lipolysis. The mediator(s) responsible for this effect remain speculative at this point. Changes in plasma concentrations of key metabolic substrates appear to be prime candidates. As already reported in several studies $^{(35,36)}$, an HFrD increases basal lactate concentrations. Our present results, collected during an exogenous lactate infusion, indicate that lactate clearance was essentially unchanged after the HFrD, and hence the observed increase in lactate concentration can be attributed to an increased lactate production. The results of the present experiment do not allow us to identify the sites of lactate production. The increased lactate production was matched by increased lactate utilisation at the whole-body level, and it is reasonable to assume that part of it was oxidised in adipose tissue and skeletal muscle. We further propose the hypothesis that increased muscle lactate uptake partially replaced lipid as an energy substrate and hence contributed to inhibit lipid oxidation. Our observation of suppressed whole-body net lipid oxidation after lactate infusion is certainly consistent with 
this hypothesis. Furthermore, we propose that increased lactate metabolism in adipose tissue may contribute to the inhibition of adipose tissue lipolysis. In support of this hypothesis, exogenous lactate infusion significantly lowered plasma NEFA in subjects fed the control diet. Lactate infusion, however, failed to further suppress plasma NEFA after the HFrD, possibly because increased adipose lactate metabolism was already maximally effective under such conditions.

The present study has several limitations that have to be acknowledged. First, compliance to the diet and fructose supplementation could not be directly assessed; however, the HFrD did cause the expected increase in plasma TAG concentrations, which indicates that the bulk of the fructose supplement had been ingested as prescribed. It remains, however, possible that fructose supplementation led to changes in fat, protein and non-fructose carbohydrate intake. Second, the HFrD provided excess fructose, total carbohydrate and energy intake. Although it is possible to speculate, based on previous reports showing an acute suppression of NEFA and a marked increase in lactate concentrations after fructose ingestion $^{(35-37)}$, that the observed inhibition of plasma NEFA was essentially linked to fructose intake, one cannot exclude that similar effects would be produced by glucose or fat overfeeding as well. Third, the volunteers of the present study were submitted to supplementation with a large fructose load, which largely exceeds usual fructose intake ${ }^{(38,39)}$. Such high sugar intakes may, however, be occasionally encountered in clinical practice. The present data nonetheless indicate that a very high fructose intake causes significant changes in adipose tissue lipolysis, and hence that fructose intake is likely to modulate the functions of adipocytes. Our previous observation, that overfeeding with half the dose of fructose as used in the present study led to a significant decrease in fasting plasma NEFA concentrations ${ }^{(7)}$, suggests that such an effect may also be present with lower, more usual fructose intake. Fourth, the $7 \mathrm{~d}$ HFrD failed to induce a significant whole-body insulin resistance in these healthy volunteers, as previously reported ${ }^{(6,7)}$. This contrasts with animal studies showing that an HFrD leads to an important reduction of insulin sensitivity, or even to overt diabetes mellitus (for a review, see Bizeau \& Pagliassotti $\left.{ }^{(40)}\right)$. Higher fructose intakes in animal studies may explain part of these differences. The duration of fructose overfeeding may also be a crucial factor, since insulin resistance was observed after several weeks of an HFrD in rodents. And finally, the calculation of exogenous lipid oxidation rests on the measurements of breath ${ }^{13} \mathrm{CO}_{2}$, which increases with an important lag time. After lipid loading, the breath ${ }^{13} \mathrm{CO}_{2}$ curve obtained after the HFrD and after the control diet started to dissociate during hour 3 of lipid loading, but the difference became significant only during hour 4 , i.e. during mental stress. We cannot, however, unequivocally attribute this difference to fructose-induced alterations of the metabolic responses to stress, since it may merely represent a carry-over effect of lipid loading. This difference nonetheless indicates that the HFrD significantly inhibited exogenous lipid oxidation, be it lower utilisation of exogenous lipids or impaired stimulation by mental stress.

In summary, the present results indicate that, in healthy human subjects, a $7 \mathrm{~d}$ supplementation with a large amount of fructose decreases whole-body lipid oxidation and hepatic ketogenesis. These effects, if present with lower, more usual, fructose intakes, may, in the long term, play an important role in the development of fructose-induced metabolic disorders. It has indeed been reported, in an animal model, that fructose-induced inhibition of hepatic lipid oxidation was directly linked to hepatic fat accumulation and hypertriacylglycerolaemia $^{(34)}$. An HFrD also stimulates lactate production, and the ensuing increase in extrahepatic lactate metabolism may contribute to impair lipid mobilisation and oxidation.

\section{Acknowledgements}

The present study was supported by a grant (310000-109737) to L. T. from the Swiss National Science Foundation.

L. T., C. B., K. A. L. and M. B. designed the study. A. A.-S. and C. B. performed the clinical studies. A. A.-S., C. B. and P. S. analysed and took part in data interpretation. K. A. L., C. B., A. A.-S. and L. T. drafted the manuscript. The authors have no conflict of interest to disclose. The authors thank Dr K. A. Acheson for having reviewed and edited the manuscript.

\section{References}

1. Bray GA, Nielsen SJ \& Popkin BM (2004) Consumption of high-fructose corn syrup in beverages may play a role in the epidemic of obesity. Am J Clin Nutr 79, 537-543.

2. Drewnowski A \& Bellisle F (2007) Liquid calories, sugar, and body weight. Am J Clin Nutr 85, 651-661.

3. Pagliassotti MJ, Prach PA, Koppenhafer TA \& Pan DA (1996) Changes in insulin action, triglycerides, and lipid composition during sucrose feeding in rats. Am J Phyisiol 271, R1319-R1326.

4. Macdonald I (1966) Influence of fructose and glucose on serum lipid levels in men and pre- and postmenopausal women. Am J Clin Nutr 18, 369-372.

5. Abraha A, Humphreys SM, Clark ML, Matthews DR \& Frayn KN (1998) Acute effect of fructose on postprandial lipaemia in diabetic and non-diabetic subjects. Br J Nutr 80, 169-175.

6. Faeh D, Minehira K, Schwarz J, Periasami R, Seongus P \& Tappy L (2005) Effect of fructose overfeeding and fish oil administration on hepatic de novo lipogenesis and insulin sensitivity in healthy males. Diabetes 54, 1907-1913.

7. Lê KA, Faeh D, Stettler R, Ith M, Kreis R, Vermathen P, Boesch C, Ravussin E \& Tappy L (2006) A 4-wk high-fructose diet alters lipid metabolism without affecting insulin sensitivity or ectopic lipids in healthy humans. Am J Clin Nutr 84, 1374-1379.

8. Chong MF, Fielding BA \& Frayn KN (2007) Mechanisms for the acute effect of fructose on postprandial lipemia. Am J Clin Nutr 85, 1511-1520.

9. Havel PJ (2005) Dietary fructose: implications for dysregulation of energy homeostasis and lipid/carbohydrate metabolism. Nutr Rev 63, 133-157.

10. Lê KA \& Tappy L (2006) Metabolic effects of fructose. Curr Opin Clin Nutr Metab Care 9, 469-475.

11. Shulman JI (2000) Cellular mechanisms of insulin resistance. $J$ Clin Invest 106, 171-176.

12. Itani SI, Ruderman NB, Schmieder F \& Boden G (2002) Lipidinduced insulin resistance in human muscle is associated with changes in diacylglycerol, protein kinase $\mathrm{C}$, and IкB- $\alpha$. Diabetes 51, 2005-2011.

13. Kelley DE, He J, Menshikova EV \& Ritov VB (2002) Dysfunction of mitochondria in human skeletal muscle in type 2 diabetes. Diabetes 51, 2944-2950. 
14. Petersen KF, Dufour S, Befroy D, Garcia R \& Shulman GI (2004) Impaired mitochondrial activity in the insulin-resistant offspring of patients with type 2 diabetes. $N$ Engl J Med 350, $664-671$.

15. Seematter G, Guenat E, Schneiter P, Cayeux C, Jéquier E \& Tappy L (2000) Effects of mental stress on insulin-mediated glucose metabolism and energy expenditure in lean and obese women. Am J Physiol 279, E799-E805.

16. Ferrannini E, Natali A, Brandi LS, Bonadonna R, De Kreutzemberg SV, DelPrato S \& Santoro D (1993) Metabolic and thermogenic effects of lactate infusion in humans. Am J Physiol 265, E504-E512.

17. Paquot N, Schneiter P, Cayeux MC, Chioléro R, Temler E, Jéquier E \& Tappy L (1995) Effects of infused sodium lactate on glucose and energy metabolism in healthy humans. Diabete Metab 21, 345-352.

18. Stunff CL \& Bougnères PF (1996) Alterations of plasma lactate and glucose metabolism in obese children. Am J Physiol 271, E814-E820.

19. Owen OE (1988) Resting metabolic requirements of men and women. Mayo Clin Proc 63, 503-510.

20. Binnert C, Pachiaudi C, Beylot M, Croset M, Cohen R, Riou JP \& Laville M (1996) Metabolic fate of an oral long-chain triglyceride load in humans. AJP Endocrinol Metab 270, E445-E450.

21. Meijssen S, Derksen RJ, Bilecen S, Erkelens DW \& Cabezas MC (2002) In vivo modulation of plasma free fatty acids in patients with familial combined hyperlipidemia using lipid-lowering medication. J Clin Endocrinol Metab 87, 1576-1580.

22. Meijssen S, Cabezas MC, Ballieux CG, Derksen RJ, Bilecen S \& Erkelens DW (2001) Insulin mediated inhibition of hormone sensitive lipase activity in vivo in relation to endogenous catecholamines in healthy subjects. J Clin Endocrinol Metab 86, 4193-4197.

23. Grouzmann E, Cavadas C, Grand D, Moratel M, Aubert JF, Brunner HR \& Mazzolai L (2003) Blood sampling methodology is crucial for precise measurement of plasma catecholamines concentrations in mice. Pflugers Arch 447, 254-258.

24. Tappy L, Dussoix P, Iynedjian P, Henry S, Schneiter P, Zahnd G, Jéquier E \& Philippe J (1997) Abnormal regulation of hepatic glucose output in maturity onset diabetes of the young caused by a specific mutation of the glucokinase gene. Diabetes 46, 204-208.

25. Rigalleau V, Binnert C, Minehira K, Stefanoni N, Schneiter P, Henchoz E, Matzinger O, Cayeux C, Jéquier E \& Tappy L (2001) In normal men, free fatty acids reduce peripheral but not splanchnic glucose uptake. Diabetes 50, 727-732.

26. DeBodo R, Steele R, Altszuler N, Dunn A \& Bishop J (1963) On the hormonal regulation of carbohydrate metabolism: studies with ${ }^{14} \mathrm{C}$-glucose. Recent Prog Horm Res 19, 445-488.

27. Tappy L, Cayeux M-C, Schneiter P, Schindler C, Temler E, Jéquier E \& Chioléro R (1995) Effects of lactate on glucose metabolism in healthy subjects and in severely injured hyperglycemic patients. Am J Physiol 268, E630-E635.

28. Livesey G \& Elia M (1988) Estimation of energy expenditure, net carbohydrate utilization, and net fat oxidation and synthesis by indirect calorimetry: evaluation of errors with special reference to the detailed composition of fuels. Am J Clin Nutr 47, 608-628.

29. Tappy L, Cayeux M-C \& Chioléro R (1996) Effects of sodium lactate on ventilation and acid-base balance in healthy humans. Clin Physiol 16, 393-401.

30. Revelly JP, Tappy L, Martinez A, Bollmann M, Cayeux MC, Berger MM \& Chioléro RL (2005) Lactate and glucose metabolism in severe sepsis and cardiogenic shock. Crit Care Med 33, 2235-2240.

31. Bantle JP, Raatz SK, Thomas W \& Georgopoulos A (2000) Effects of dietary fructose on plasma lipids in healthy subjects. Am J Clin Nutr 72, 1128-1134.

32. Tappy L, Paquot N, Tounian P, Schneiter P \& Jéquier E (1995) Assessment of glucose metabolism in humans with the simultaneous use of indirect calorimetry and tracer techniques. Clin Physiol 15, 1-12.

33. Ji H \& Friedman M (2007) Reduced capacity for fatty acid oxidation in rats with inherited susceptibility to diet-induced obesity. Metabolism 56, 1124-1130.

34. Roglans N, Vila L, Farre M, Alegret M, Sanchez RM, VazquezCarrera M \& Laguna JC (2007) Impairment of hepatic Stat-3 activation and reduction of PPAR $\alpha$ activity in fructose-fed rats. Hepatology 45, 778-788.

35. Tounian P, Schneiter P, Henry S, Jéquier E \& Tappy L (1994) Effects of infused fructose on endogenous glucose production, gluconeogenesis and glycogen metabolism in healthy humans. Am J Physiol 267, E710-E717.

36. Teff KL, Elliott SS, Tschöp M, Kieffer TJ, Rader D, Heiman M, Townsend RR, Keim NL, D'Alessio DA \& Havel PJ (2004) Dietary fructose reduces circulating insulin and leptin, attenuates postprandial suppression of ghrelin, and increases triglycerides in women. J Clin Endocrinol Metab 89, 2963-2972.

37. Tappy L, Randin JP, Felber JP, Chioléro R, Simonson DC, Jéquier E \& DeFronzo RA (1986) Comparison of thermogenic effect of fructose and glucose in normal humans. Am J Physiol 250, E718-E724.

38. Johnson RJ, Segal MS, Sautin Y, Nakagawa T, Feig DI, Kang DH, Gersch MS, Benner S \& Sanchez-Lozada LG (2007) Potential role of sugar (fructose) in the epidemic of hypertension, obesity and the metabolic syndrome, diabetes, kidney disease, and cardiovascular disease. Am J Clin Nutr 86, 899-906.

39. Montonen J, Jarvinen R, Knekt P, Heliovaara M \& Reunanen A (2007) Consumption of sweetened beverages and intakes of fructose and glucose predict type 2 diabetes occurrence. J Nutr 137, 1447-1454.

40. Bizeau ME \& Pagliassotti MJ (2005) Hepatic adaptations to sucrose and fructose. Metab Clin Exp 54, 1189-1201. 